\title{
NOTCH2NLA silencing inhibits ovarian carcinoma progression and oncogenic activity in vivo and in vitro
}

\author{
Wendi Guo $^{1 \wedge}$, Zhen Sun ${ }^{1}$, Nannan Zhao ${ }^{1}$, Yiwen Zhou ${ }^{2}, J_{i e}$ Ren $^{1}$, Lingling Huang ${ }^{1}$, Yi Ping $^{1 \wedge}$ \\ ${ }^{1}$ Department of Obstetrics and Gynecology, Second Hospital of Shanxi Medical University, Taiyuan, China; ${ }^{2}$ Department of Obstetrics and \\ Gynecology, First Affiliated Hospital of University of South China, Hengyang, China \\ Contributions: (I) Conception and design: Y Ping, W Guo, Z Sun; (II) Administrative support: Y Ping; (III) Provision of study materials or patients: W \\ Guo, Z Sun; (IV) Collection and assembly of data: W Guo, N Zhao; (V) Data analysis and interpretation: Y Zhou, L Huang, J Ren; (VI) Manuscript \\ writing: All authors; (VII) Final approval of manuscript: All authors. \\ Correspondence to: Yi Ping. Department of Obstetrics and Gynecology, Second Hospital of Shanxi Medical University, No. 382 Wuyi Road, Taiyuan \\ 030000, China. Email: pingyi7110@126.com.
}

Background: Among all gynecological malignancies, ovarian cancer (OC) is prone to recurrence, metastasis, and resistance, and has the highest mortality rate. Furthermore, China is about to face the risk of increased incidence of the disease. This study aimed to identify potential novel tumorigenesis and prognostic genes in OC.

Methods: The Genotype-Tissue Expression (GTEx) project and The Cancer Genome Atlas (TCGA) project were used to obtain mRNA sequencing datasets and clinical information to identify candidate genes critical to OC carcinogenesis and progression. The potential genes affecting OC were performed by comprehensive survival analysis, followed by external validation. Cell proliferation, wound healing, and transwell assays validated the effects of a potential gene on proliferation, migration, and invasion in the A2780 cell line. Observe xenograft formation in nude mice and use immunohistochemistry to determine the expression of epithelial-mesenchymal transition (EMT)-related proteins in xenograft tumors after gene silencing. Two-tailed Student's $t$-tests were used for two-group comparisons in in vivo and in vitro experiments.

Results: The current study developed an eight-gene model for predicting the survival of patients with OC, in which PI3, CCDC80, IL27RA, HERC1, and NOTCH2NLA represented adverse, HMGB3, CXCL11, and $C X C L 9$ represented protective prognosis. Further, repression of NOTCH2NLA, the selected potential gene, blocked the proliferation, migration, and invasion of A2780 cells in vitro and inhibited the growth of xenograft tumors in vivo. Finally, we revealed that the expression of NOTCH2NLA in OC negatively correlated with that of E-cadherin and positively correlated with that of vimentin and SNAI2.

Conclusions: The study provided a novel view on the biological significance and function of NOTCH2NLA and confirmed that NOTCH2NLA plays a significant role in OC prognosis by affecting EMT, which could be used as a new biomarker for OC prognosis.

Keywords: Ovarian cancer (OC); integrated bioinformatics analysis; epithelial-mesenchymal transition (EMT); prognosis; biomarkers

Submitted Sep 10, 2021. Accepted for publication Nov 10, 2021.

doi: $10.21037 /$ atm-21-5435

View this article at: https://dx.doi.org/10.21037/atm-21-5435

^ ORCID: Wendi Guo, 0000-0001-6479-6341; Yi Ping, 0000-0003-2757-9465. 


\section{Introduction}

Advanced ovarian cancer (OC) patients have benefited significantly from targeted therapy with poly (ADP-ribose) polymerase inhibitors (PARPi) and bevacizumab, achieving prolonged progression-free survival in recent years. However, the mortality rate of OC has shown an upward trend both in China and other countries (1-3), consistently ranking first among all gynecological malignancies (4). China is currently at risk of facing an increased incidence of OC because countries with a high Human Development Index have a higher incidence according to GLOBOCAN 2020 (1). Therefore, it is necessary to further identify new targets affecting the carcinogenesis and progression of OC.

Epithelial-mesenchymal transition (EMT) is known to contribute to tumor metastasis by enhancing mobility, invasion, self-renewal, and resistance to apoptosis. Mesenchymal markers such as snail family transcriptional repressor 2 (SNAI2) are generally positively correlated with neurogenic locus notch homolog (NOTCH) proteins (5) and are closely related to the progression of OC. Similarly, the activation of the Notch pathway can promote malignant transformation and metastasis of OC by mediating EMT (6), promoting cell proliferation (7), expanding drug-resistant stem cells (8), participating in angiogenesis (9), and affecting T cell immunity (10).

Notch $2 \mathrm{~N}$-terminal like (NOTCH2NL) is highly similar to Notch receptor 2 (NOTCH2) in structure and expression patterns in humans and enhances the ability of the Notch pathway to affect the development of human cortical organs (11). NOTCH2NL has been confirmed as having high-frequency mutations in anaplastic astrocytoma (12) and brain metastases of non-small cell lung cancer (NSCLC) (13), and focal and broad gain of NOTCH2NL has been frequently observed in anaplastic thyroid cancer (ATC) (14). While these findings suggest the significance of NOTCH2NL in malignant tumors, its role in gynecological tumors has not been explored, and whether its expression affects OC development caused by EMT remains unclear.

The main aim of this study was to use integrated bioinformatics to analyze genes that may affect the prognosis of OC and to establish a prognostic gene model. Furthermore, we used in vivo and in vitro experiments to examine whether Notch $2 \mathrm{~N}$-terminal like A (NOTCH2NLA) could mediate EMT and affect the progression of OC. A protocol was prepared before the study without registration. We present the following article in accordance with the ARRIVE reporting checklist (available at https://dx.doi.org/10.21037/atm-21-5435).

\section{Methods}

\section{Data collection and screening of differentially expressed genes (DEGs)}

OC RNAseq microarray gene expression data and the clinical information of 379 OC samples from The Cancer Genome Atlas (TCGA) database, combined with clinical information of 88 normal samples from the GenotypeTissue Expression (GTEx) database, were downloaded. The gene expression profile data of 111 patients with OC downloaded from the International Cancer Genome Consortium (ICGC) were also utilized. The study was conducted in accordance with the Declaration of Helsinki (as revised in 2013) and the limma package of $\mathrm{R}$ software was used for merging, data correction, and analysis.

\section{Functional enrichment and gene set enrichment analyses (GSEA)}

The ClusterProfiler of $\mathrm{R}$ software was used to conduct Gene Ontology (GO) analysis and Kyoto Encyclopedia of Genes and Genomes (KEGG) pathway analysis of the DEGs functions. We set "c2.cp.kegg.v7.0.symbols.gmt" and "c5.all. v7.0.symbol.gmt" as the gene set database to further screen pathways of DEGs between the normal group and OC group.

\section{Protein-protein interaction (PPI) networking and hub gene analysis}

The Search Tool for the Retrieval of Interacting Genes (STRING) (version 10.0) was used to construct the PPI network of the DEGs. The PPI network was then analyzed using Cytoscape (version 3.4.0) software, and the top 10 hub genes were classified using cytohubba.

\section{Tumor microenvironment analysis and survival analysis}

DEGs were subsequently used for Kaplan-Meier (K-M) analysis and univariate Cox analysis to identify potential prognostic genes for OC. Lasso regression further assessed the impact of these prognostic genes on patient outcomes. The patients were divided into high-risk and low-risk groups based on the median risk score, and a multivariate Cox regression model was applied using $\mathrm{R}$ software survival and survminer packages. The accuracy of the risk scoring 
model for predicting overall prognosis was analyzed using the survival receiver operating characteristic (ROC). Finally, comprehensive survival analysis was performed on OC at different stages.

\section{Cell culture}

A2780-GFP cells (ATCC, USA) were cultured in a humid environment with $5 \% \mathrm{CO}_{2}$ at $37^{\circ} \mathrm{C}$, and $\mathrm{RPMI}$ 1640 medium (Thermo Fisher Scientific, USA) was supplemented with $10 \%$ fetal bovine serum (FBS) (Thermo Fisher Scientific, USA), $100 \mathrm{mg} / \mathrm{mL}$ streptomycin and $100 \mathrm{U} / \mathrm{mL}$ penicillin.

\section{Small interfering RNA (siRNA) preparation and cell transfection}

Two different siRNAs duplexes targeting NOTCH2NLA sequences and a negative control siRNA were commercially designed and synthesized by GenePharma (China). The sequences were as follows: NOTCH2NLA siRNA1, sense: 5'-GGCACAGGAUACUGCAAAUTT-3'; and anti-sense: 5'-AUUUGCAGUAUCCUGUGCCTT-3'; NOTCH2NLA siRNA2, sense: 5'-CCCUGUGCAAAUGGAAGUATT-3'; and anti-sense: 5'-UACUUCCAUUUGCACAGGGTT-3'. $\mathrm{Neg}$ a tive control si R A, s e n s e : 5'-UUCUCCGAACGUGUCACGUTT-3'; anti-sense: 5'-ACGUGACACGUUCGGAGAATT-3'. A2780-GFP cells $\left(4 \times 10^{5}\right)$ were seeded in 6 -well plates overnight and cultured in serum-free medium for $6 \mathrm{~h}$ once reaching $70-90 \%$ confluency. The diluted $0.15 \mathrm{nmol}$ siRNA/3.75 $\mu \mathrm{L}$ Lipofectamine $^{\mathrm{TM}} 2000$ (Thermo Fisher Scientific, USA) complex was added per well for $5 \mathrm{~h}$ according to the manufacturer's protocol, after which standard cell culture medium replaced the complex for $24 \mathrm{~h}$. All assays performed with transfected cells were completed within $24-48 \mathrm{~h}$ after transfection.

\section{Wound healing assay}

A 2-mm wide plastic pipette tip was used to make a scratch when A2780-GFP cells transfected with NOTCH2NLA siRNA or negative control siRNA were grown to $80-90 \%$ confluence in a 96-well plate. Cells were washed three times with phosphate buffered saline then cultured in complete medium for $24 \mathrm{~h}$ and the cell migration ability observed using the Lionheart ${ }^{\mathrm{TM}} \mathrm{FX}$ automatic live cell imager (BioTek Instruments, USA). The migration rate $=($ the area covered by the cells at $24 \mathrm{~h}$ - the area covered by the cells at $0 \mathrm{~h}$ )/ the area covered by the cells at $0 \mathrm{~h}$.

\section{Transwell assay}

The perforated filters $(8 \mu \mathrm{m})$ covered with Matrigel (BD Biosciences, USA) were inserted into 24-well plates (SigmaAldrich, GER). A2780-GFP cells with NOTCH2NLA siRNA or negative control siRNA were resuspended in $250 \mu \mathrm{L}$ RPMI 1640 medium without FBS in each upper chamber, and $450 \mu \mathrm{L}$ RPMI 1640 medium with $30 \%$ FBS was added to each lower compartment. The cells were incubated at $37{ }^{\circ} \mathrm{C}$ in a 24 -well plate for $24 \mathrm{~h}$, and Lionheart ${ }^{\mathrm{TM}} \mathrm{FX}$ was used to count cells and automatically capture fluorescent images hourly.

\section{Survival assay using automated live cell imaging}

A2780-GFP cells were cultured in the presence of NOTCH2NLA siRNA or negative control siRNA for $24 \mathrm{~h}$ then seeded into a 96 -well plate at a density of $5 \times 10^{3}$ cells per well. Lionheart ${ }^{\mathrm{TM}} \mathrm{FX}$ was used to acquire the phase images at each time point.

\section{Purchase and breeding of animals}

Six-week-old NU/NU female nude mice (19-23 g/per mouse) with deficient immunity were obtained from Charles River Laboratory (China). They were maintained in a specific pathogen-free "barrier" facility and housed under controlled temperature and humidity and alternating 12-hour light and dark cycles. The female mice received specific pathogen-free mouse chow and were allowed to drink sterile water ad libitum. Experiments were performed under a project license (No. SYDL2021007) granted by the animal ethics board of Shanxi Medical University, in compliance with the Ministry of Science and Technology of China guidelines for the care and use of animals.

\section{Establishment of subcutaneous xenograft model}

A total of 12 nude mice were included, with three mice in each group and the experiment repeated twice. Mice were randomly implanted subcutaneously in the left armpit region with $300 \mu \mathrm{L}$ serum-free medium containing either A2780/NOTCH2NLA siRNA or A2780/negative siRNA $\left(1 \times 10^{6}\right.$ cells in either case), then sacrificed for the resection of xenografts after six weeks. The xenograft sizes were 
measured, and the tumor volumes calculated using the formula for a scalene ellipsoid (units of cubic centimeters): height $\times$ width $\times$ length $\times 0.52$.

\section{Hematoxylin and eosin (HE) staining}

Tumor tissues of mice were fixed with paraformaldehyde, dehydrated using ethanol solutions, embedded in paraffin, sliced into sections every $100 \mu \mathrm{m}$, then stained with HE. Tissue sections were scanned using a Pannoramic MIDI digital slide scanner (3DHISTECH, Ltd., Hungary) to detect morphological differences in tumor cells, the area of tumor necrosis, and the number of necrotic foci.

\section{Immunobistochemistry (IHC) and scoring}

The tissue sections $(5 \mu \mathrm{m})$ were deparaffinized, quenched with $3 \%$ hydrogen peroxide, antigen retrieved with EDTA, and incubated with $10 \%$ goat serum, then combined with the primary antibody 12129-1-AP (1:100), 20874-1-AP (1:100), 10366-1-AP (1:300), 12129-1-AP (1:300) (Proteintech, China) at $4{ }^{\circ} \mathrm{C}$ overnight. Sections were then incubated with secondary antibody ab205718 (1:2,000) (Abcam, UK) at $37^{\circ} \mathrm{C}$ for $1 \mathrm{~h}$, then visualized using EnVision ${ }^{\mathrm{TM}}$ Detection Systems (Dako, Denmark). The immunohistochemical staining intensity and area of Ki67, E-cadherin, vimentin, and SNAI2 were semi-quantitatively assessed using ImagePro Plus 6.0 (Media Cybernetics, USA).

\section{Statistical analysis}

Each vitro experiment was performed on three independent occasions. Data are presented as the mean \pm SD. Statistical analyses were performed using SPSS (version 26.0; IBM, USA) and GraphPad Prism 8 (GraphPad, USA), and two-tailed Student's $t$-tests were used for two-group comparisons. Differences were considered statistically significant at $\mathrm{P}<0.05$.

\section{Results}

\section{DEGs, GO, and KEGG}

A total of 2,253 DEGs were found in the tumor and normal ovarian tissues, and of these, 1,017 were upregulated and 1,236 were downregulated (shown in Figure 1A).
DEGs were primarily involved in immunoglobulin regeneration, regulation of humoral immune response, cell junction organization, cell-cell junction adhesion, and extracellular matrix (shown in Figure 1B). In signaling pathway enrichment analysis, DEGs were associated with cell adhesion molecules, T helper 1 and $\mathrm{T}$ helper 2 cell differentiation, cell cycle, protein 53 signaling pathway, and the RAS signaling pathway (shown in Figure 1C).

\section{Hub gene and miRNA expression profiles}

In the network, the top 10 node genes were ribosomal protein (RP) L27A, RPL31, RPL34, RPL37A, RPL23, RPL26, RPL22, RPL39, RPL30, and RPS27A, and were each considered hub genes (shown in Figure 2A). Among them, RPS27A was associated with EMT mediated by the Notch pathway (shown in Figure 2B).

In the univariate Cox regression analysis, 228 DEGs were significantly associated with overall survival (OS). The multivariate Cox regression analysis constructed after Lasso regression analysis comprised eight DEGs identified as significant prognostic factors for OS (shown in Figure 2C), among which, NOTCH2NLA had the highest hazard radio among the eight genes.

\section{Survival analysis of the low-risk and high-risk OC groups}

In the risk scoring model, 187 and 188 patients were in the high-risk and low-risk groups, respectively. Survival analysis showed that the OS rate of the low-risk group was higher than that of the high-risk group (shown in Figure $3 A$ ). ROC analysis of the risk score model based on the eight DEGs identified as prognostic factors showed excellent execution in survival prediction, with an area under the curve (AUC) of $0.700,0.715$, and 0.770 for the 1-, 3-, and 5-year OS, respectively. The results suggest that in the tumor microenvironment, the eight genes may be closely related to OC stromal cells (shown in Figure 3B).

The eight-gene model had a poor predictive value in stages I and II $(\mathrm{P}=0.812)$ but a higher value for stages III and IV (shown in Figure 3C). ROC analysis showed that the risk score had a good predictive ability for the 1-, 3-, and 5 -year OS in the ICGC OC dataset, and the AUC was 0.6. Analysis showed that the expression levels of the eight genes were different in OC tissue and normal ovarian tissue, lowrisk, and high-risk groups (shown in Figure 3D). 

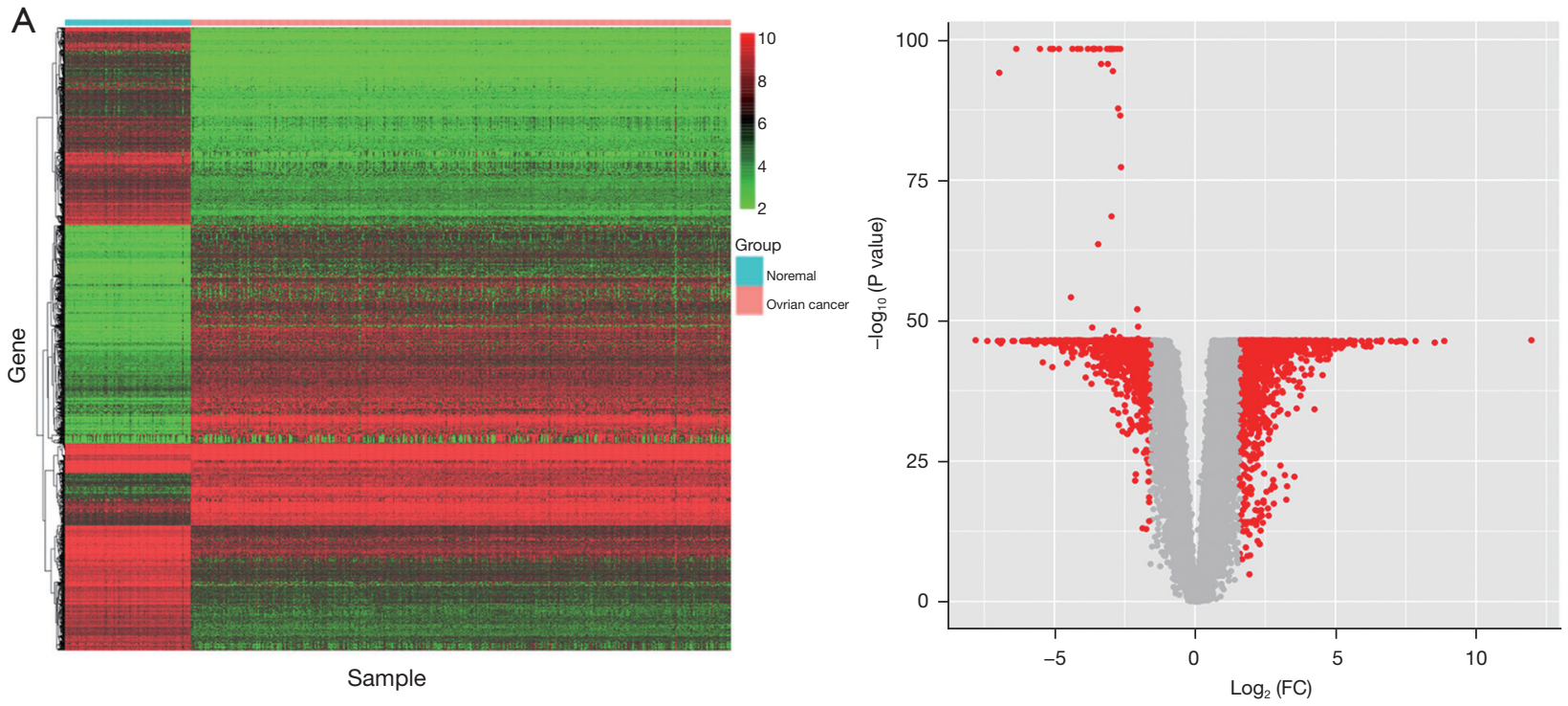

B

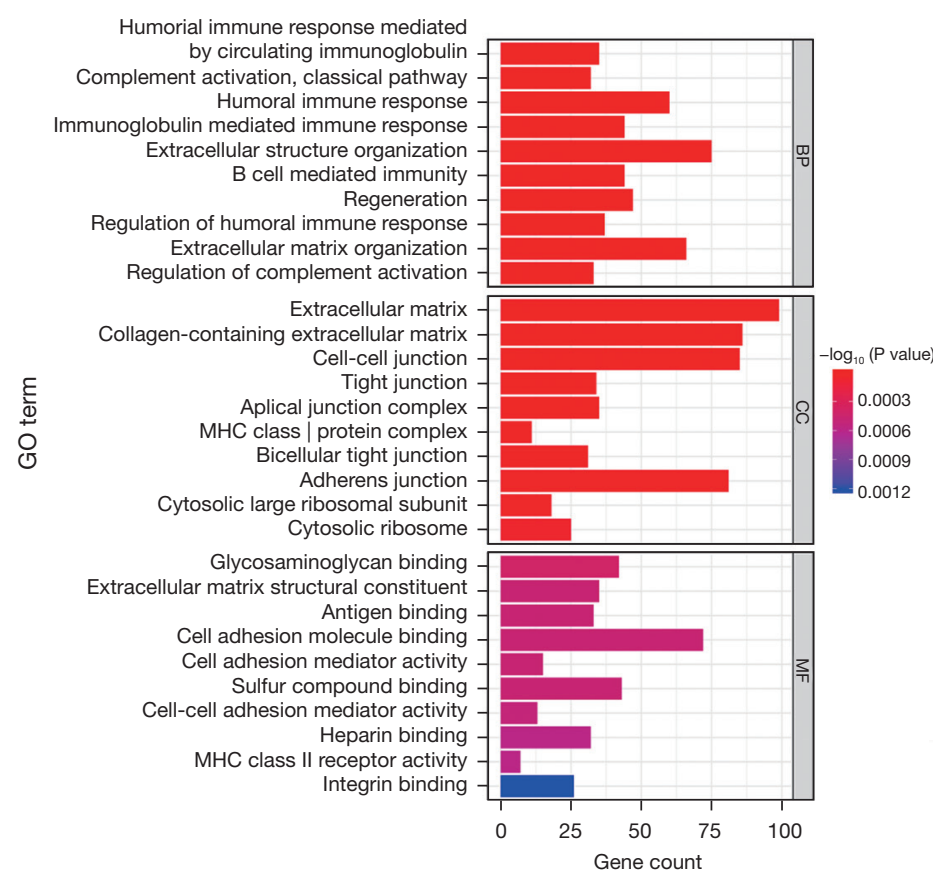

C

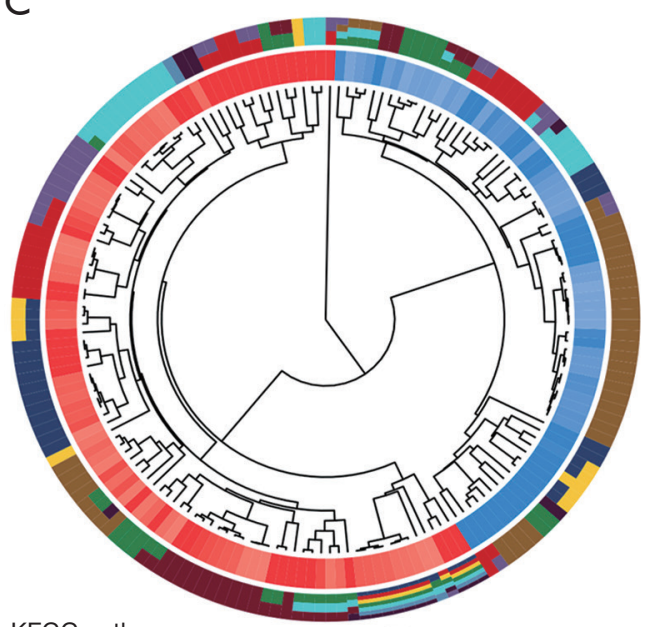

KEGG pathways

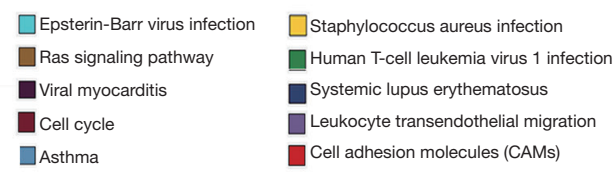

Figure 1 Screening and analysis of DEGs. (A) Heat map of DEGs in TCGA and GTEx datasets (left) and Volcanic map (right). The gradient from green to red indicates a change in gene expression from downregulation to upregulation. Red nodes represent DEGs with $\mid \log 2 \mathrm{FCl} \geq 1$ and FDR $<0.05$. (B) GO enrichment analysis of DEGs. ClusterProfiler annotated 2,253 DEGs for GO functional analysis of BP, CC, and MF in biological processes. The listed genes were significantly enriched $(\mathrm{P}<0.05)$. (C) KEGG Circle plot: the outer circle shows a scatter plot of the rhythmically expressed tumor-associated gene levels of each enriched KEGG term. The red circle indicates up, and the blue circle indicates down. The inner ring is a bar graph in which the height of the bar indicates the significance of the KEGG term (the $\log$ value of the $\log 10$ adjustment), and the color corresponds to the z score: blue, decreased; red, increased; and white, unchanged. DEG, differentially expressed gene; TCGA, The Cloud Genome Atlas; GTEx, Genotype-Tissue Expression; FC, fold change; FDR, false discovery rate; GO, Gene Ontology; BP, biological process; CC, cellular component; MF, molecular function; KEGG, Kyoto Encyclopedia of Genes and Genomes. 

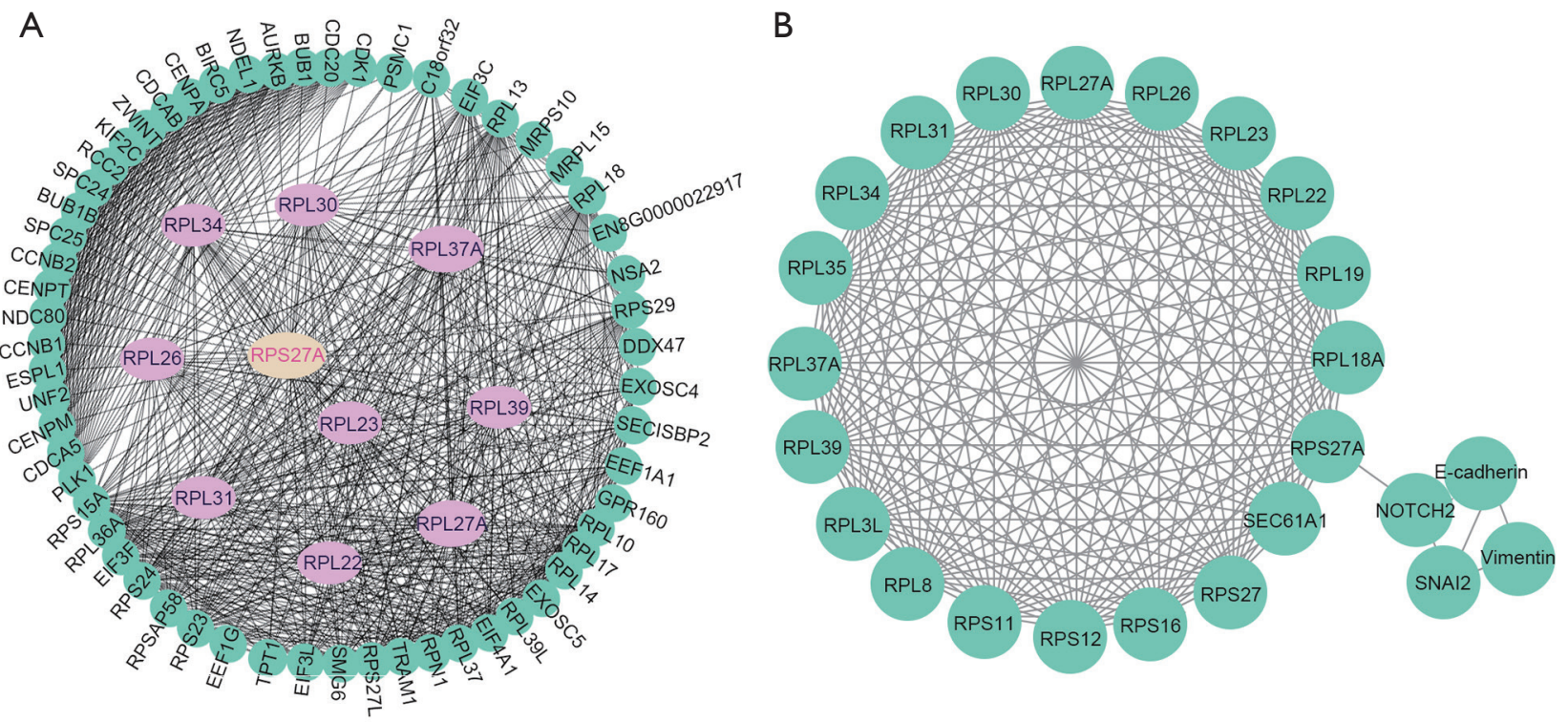

C

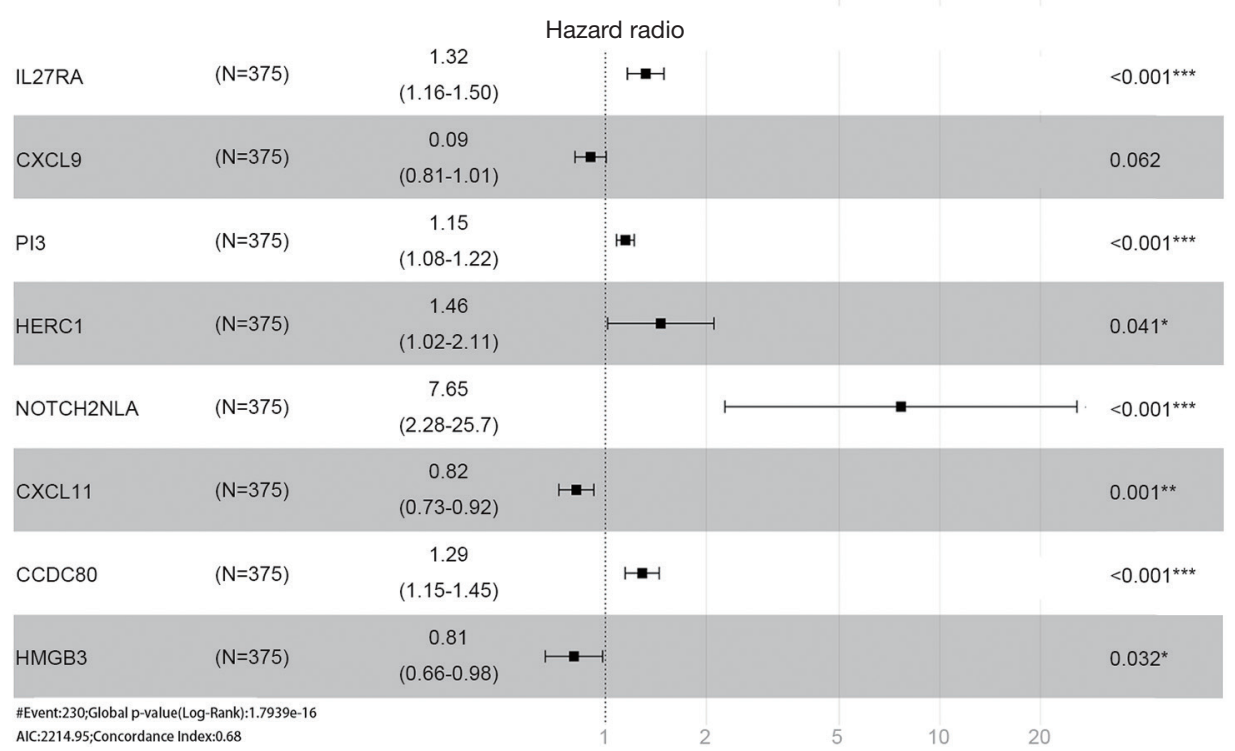

Figure 2 The interaction network and biological process of central genes, and the establishment and survival analysis for eight genes. (A) The module consisting of 10 nodes identified by cytohubba. (B) Ten hub genes related to each other, among which RPS27A is associated with EMT mediated by the Notch pathway. (C) Prognostic value of eight genes in OC patients of the TCGA. *, P<0.05; **, P<0.01; ***, $\mathrm{P}<0.001$. EMT, epithelial-mesenchymal transition; OC, ovarian cancer; TCGA, The Cloud Genome Atlas.

\section{NOTCH2NLA silencing inbibits cell migration, invasion, and proliferation}

A2780-GFP cells transfected with NOTCH2NLA siRNA showed significantly decreased wound healing and invasion capacity compared to those transfected with negative control siRNA (shown in Figure 4A,4B). Similarly, the viability of A2780-GFP cells transfected with NOTCH2NLA
siRNA was significantly reduced compared with that of cells transfected with the negative control siRNA during the 12 and $24 \mathrm{~h}$ recorded by Lionheart ${ }^{\mathrm{TM}} \mathrm{FX}$ (shown in Figure $4 C$ ).

\section{NOTCH2NLA silencing inbibits xenograft growth and EMT in vivo}

Our data showed that NOTCH2NLA silencing in A2780- 
A

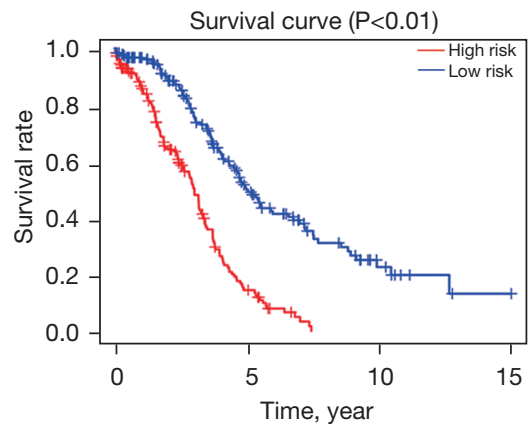

C

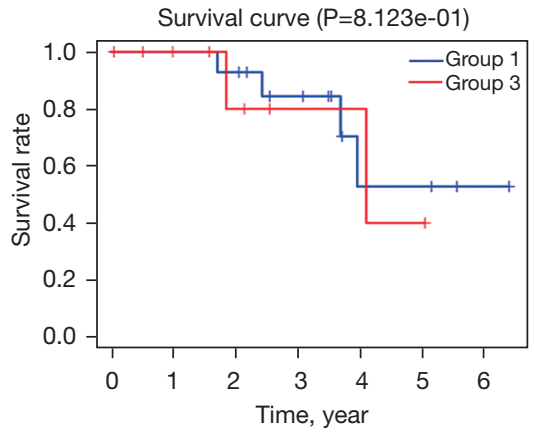

B
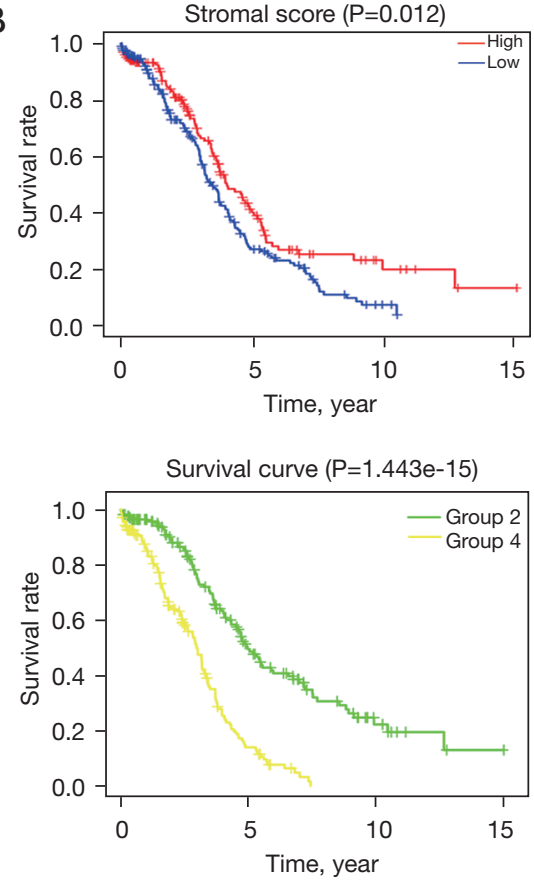

D
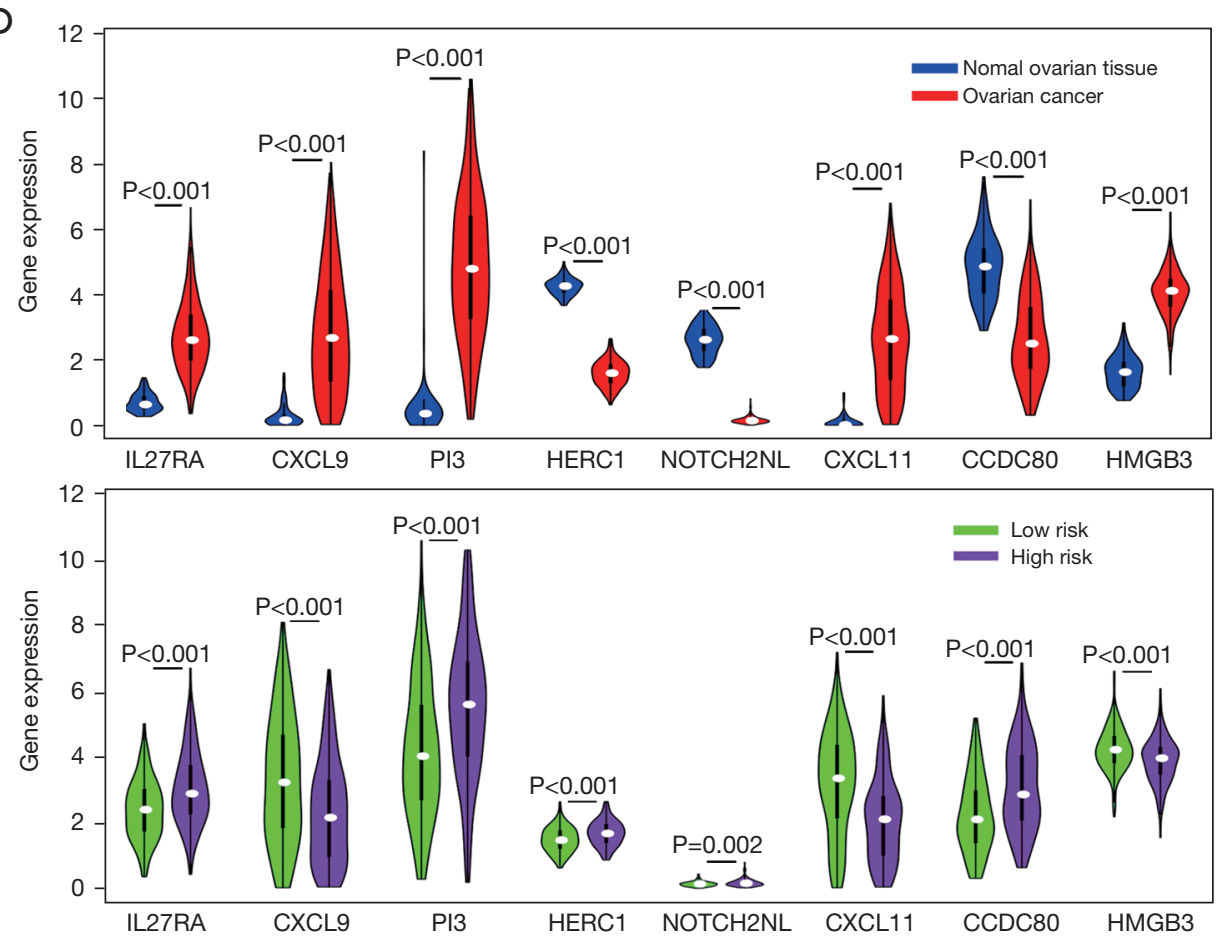

Figure 3 Survival analysis with the low-risk and high-risk OC groups. (A) Survival curves for low-risk and high-risk groups. (B) In the tumor microenvironment, ESTIMATE analysis revealed that eight genes were mainly associated with OC stromal cells. (C) The eight-gene model has a high predictive value for advanced OC. Group 1: low risk and stage I + II; Group 2: low risk and stage III + IV; Group 3: high risk and stage I + II; and Group 4: high risk and stage III + IV. (D) Expression of the eight genes in the TCGA OC dataset. OC, ovarian cancer; TCGA, The Cancer Genome Atlas. 

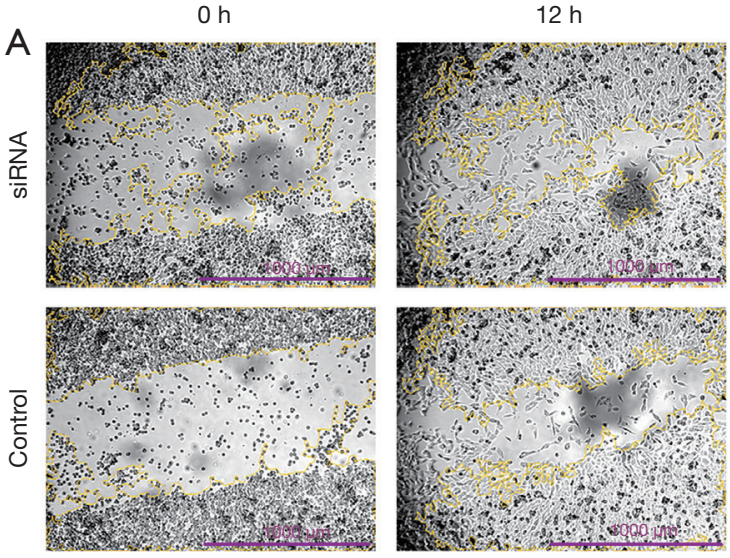

$\mathrm{Oh}$
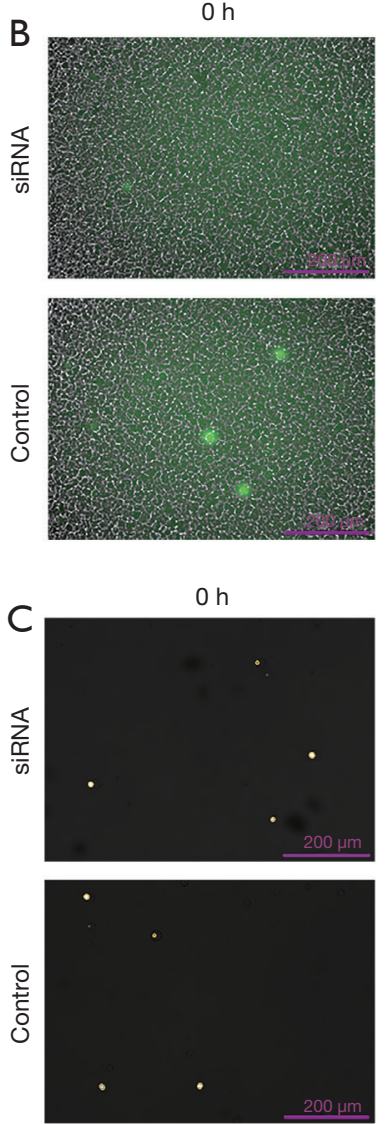

$12 \mathrm{~h}$
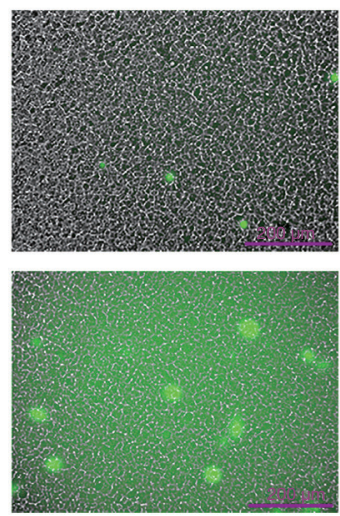

$12 \mathrm{~h}$
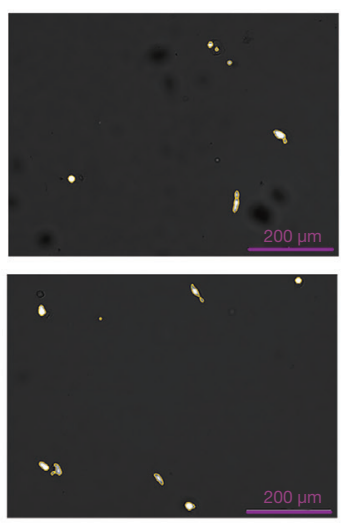

$24 \mathrm{~h}$
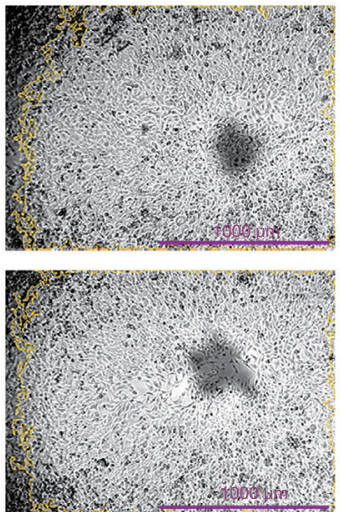

$24 \mathrm{~h}$
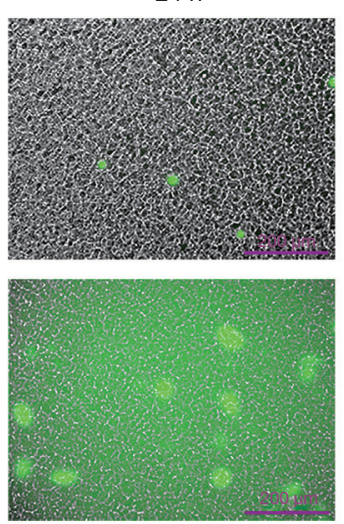

$24 \mathrm{~h}$
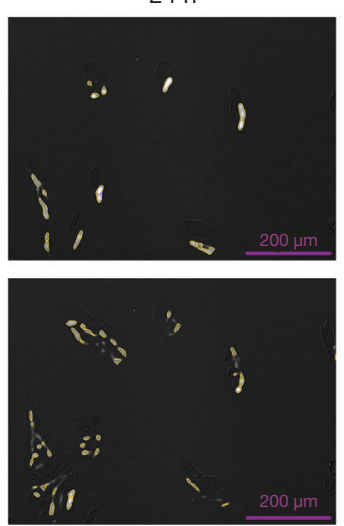
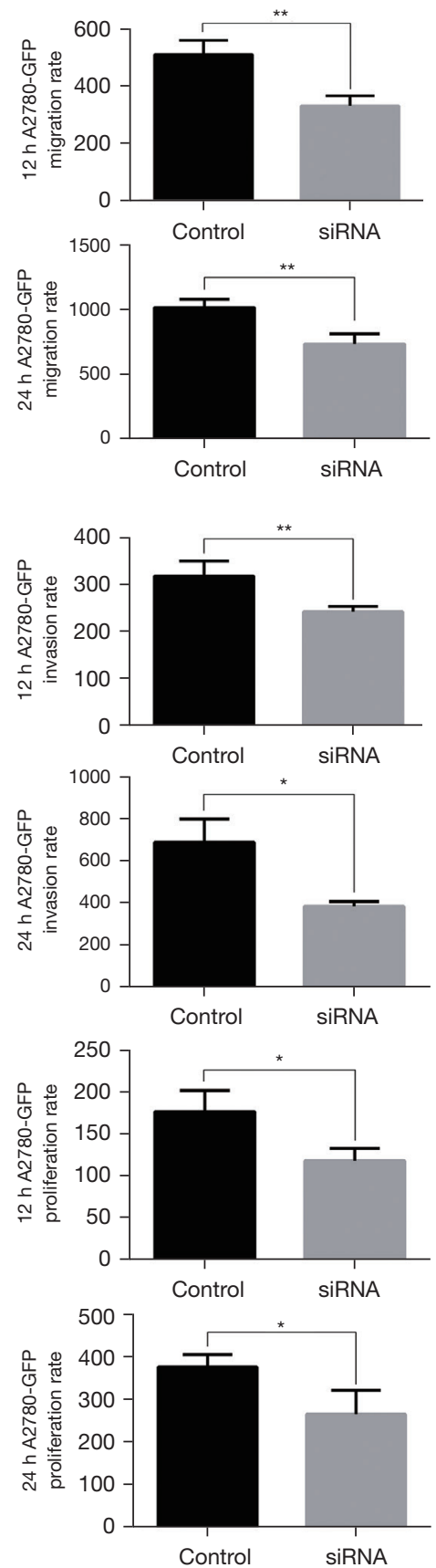

Figure 4 NOTCH2NLA silencing inhibited the migration, invasion, and proliferation of A2780-GFP cells. (A) Representative pictures (left) from tumor cell wound healing assays. The graph (right) is the quantified data of the assay (n=6). Scale is 1,000 $\mu \mathrm{m}$. (B) The invasion of A2780-GFP cells as determined using transwell assays with $8 \mu \mathrm{m}$ pores in polycarbonate membranes. Representative images (left) and quantification (right) of A2780-GFP cell invasion ( $\mathrm{n}=6$ ). Scale is $200 \mu \mathrm{m}$. (C) A living cell imaging system recorded representative images (left) and measured quantitative proliferation (right) of A2780-GFP cells. Scale is $200 \mu \mathrm{m}$. Bars indicate mean $\pm \mathrm{SD}(\mathrm{n}=6) .{ }^{*}, \mathrm{P}<0.05 ;{ }^{* *}, \mathrm{P}<0.01$. 
GFP cells suppressed xenograft growth (shown in Figure $5 A$ ) and $\mathrm{HE}$ staining showed a larger necrotic area and more necrotic foci of the xenografts in the control group than in the NOTCH2NLA-silenced group (shown in Figure 5B).

Immunohistochemical analysis of xenografts showed that the expression of Ki67, vimentin, and SNAI2 was significantly lower in the NOTCH2NLA-silenced group than in the negative control group, whereas E-cadherin expression was increased (shown in Figure 5C). This suggested NOTCH2NLA promotes EMT progression.

\section{Discussion}

The mortality rate of OC is the highest among all gynecological malignancies and is rising. To date, complete/ partial remission is difficult to maintain, and new directions affecting the carcinogenesis and progression of the disease must be identified. Here, we used integrated bioinformatics analysis to explore potential biomarkers related to the prognosis of OC and developed an eight-gene model for predicting patient survival which was proven to have good predictive value. NOTCH2NLA had a high predictive value despite its low expression level. The results indicated that NOTCH2NLA silencing in OC cell lines could inhibit the migration and invasion of malignant cells and promote apoptosis in vitro. Consistent with this, NOTCH2NLA silencing inhibited mitosis in mouse xenografts in vivo, and its downregulation inhibited the proliferation of OC cells. In addition, this study confirmed that it promotes EMT progression. Altogether, these data confirmed that NOTCH2NLA plays a role in OC progression.

\section{Eight-gene prognostic model}

The high predictive value of the eight-gene model for stage III and IV may suggests these genes, rather than acting as oncogenic drivers, are more critical in the progression and metastasis of OC. Among the genes in the model, HECT And RLD domain containing E3 ubiquitin protein ligase family member 1 (HERC1) (15), interleukin 27 receptor subunit alpha (IL27RA) (16), coiled-coil domain containing 80 (CCDC80) (17), and high mobility group box 3 (HMGB3) (18) may act as tumor suppressors or oncogenes in specific cancer types, while C-X-C motif chemokine 9 (CXCL9), C-X-C motif chemokine 11 (CXCL11), and peptidase inhibitor 3 (PI3) are closely related to OS in OC.
Similar to our study, Millstein et al. (19) and Yan et al. (20) used bioinformatics analysis to confirm CXCL9 and CXCL11 were associated with better OS in OC patients, respectively. Both CXCL9 and CXCL11 could recruit T and B lymphocytes and natural killer cells to inhibit tumor growth, and the expression of CXCL11 was closely related to antigen-related genes, immune checkpoint-related genes, and PARPi therapy (21). However, a previous study showed that PI3 was highly expressed in the high-risk group of OC (22), which is inconsistent with our results and requires further experimental confirmation. Apart from the nervous system, NOTCH2NL has only been reported in NSCLC, ATC, and Mayer-Rokitansky-Küster-Hauser syndrome $(13,14,23)$. Here, we found that its expression was higher in normal ovarian tissues than in OC tissues, and that the lower the expression of NOTCH2NL, the better the prognosis in OC, reflecting the complexity of molecular involvement in tumorigenesis. However, our results on NOTCH2NL expression and prognosis require further confirmation.

\section{New perspective on NOTCH2NL}

Previous studies have used exome sequencing to confirm high-frequency mutations in NOTCH2NL in various tumors (12-14), and somatic mutations and copy number gain of NOTCH2NL are potential oncogenic events in tumorigenesis. Our cell assays confirmed that NOTCH2NLA could promote proliferation, migration, and invasion in $\mathrm{OC}$, indicating its significance is not limited to neurology. A previous study showed that the knockdown of NOTCH2NL in the glioma HSJD002 cell line also significantly reduced cell numbers (24), which is consistent with our results.

Differentiation and proliferation in the ventral forebrain reflect the region-specific patterning of NOTCH2NL expression (24). The ovaries have the most types of primary tumors of all body organs, among which germ cell tumors contain 2-3 germ layers. Immature ovarian teratomas are composed of immature embryonic tissues with different degrees of differentiation but mainly primitive neural tissues derived from the ectoderm, suggesting that NOTCH2NL may be involved in the occurrence and development of $\mathrm{OC}$ and has tissue specificity. Therefore, whether the carcinogenic function of NOTCH2NL in A2780 cells is a cell type-specific effect or shared by multiple OC cell lines 


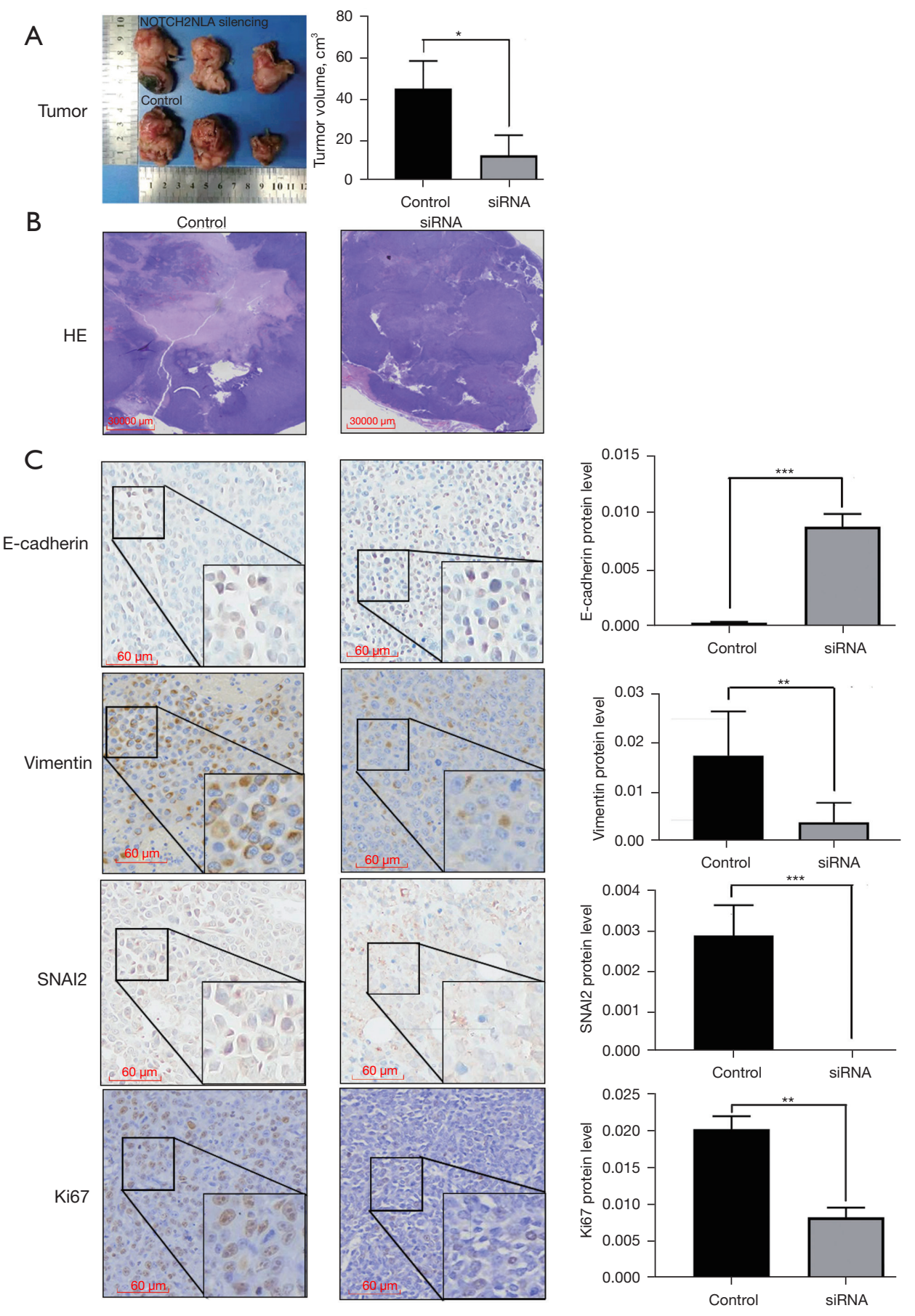

Figure 5 In vivo, NOTCH2NLA silencing inhibited tumor growth and EMT-related protein expression. (A) NOTCH2NLA-silenced suppression of mouse A2780-GFP cell xenograft growth in vivo. The size of the xenografts was compared between the transfection and negative control groups under the ruler (left). Quantification (right) of tumor volume between the NOTCH2NLA transfection and negative control groups (n=3). (B) HE staining of xenografts observed under the microscope. (C) Immunohistochemical analysis of the expression of EMT-associated proteins and Ki67 protein. The graph (right) represents quantified data of the immunohistochemical analysis (n=6). *, $\mathrm{P}<0.05$; **, $\mathrm{P}<0.01$; ***, $\mathrm{P}<0.001$. HE, hematoxylin and eosin; EMT, epithelial-mesenchymal transition. 
remains to be elucidated.

\section{NOTCH2NLA-mediated EMT}

Combined with tumorigenicity in vivo, using immunohistochemical semi-quantitative analysis we found that the expression of NOTCH2NLA in OC is negatively correlated with that of E-cadherin and positively correlated with that of vimentin and SNAI2, proving that the regulation of EMT-related proteins by NOTCH2NLA might be a critical factor in OC metastasis. Huang et al. (6) recently found that the Notch ligand Delta-like 1 (DLL1) promotes EMT in ovarian high-grade serous carcinoma via activation of the Notch pathway. NOTCH2NL genes are mutated synchronously with NOTCH2 in NSCLC, and their proteins increase clonal expansion and enhance Notch signaling by interacting with the NOTCH receptor, even though previous studies have confirmed that NOTCH2NL represses the transcriptional activities of the NOTCH intracellular domain and affects the function and trafficking of DLL1 (25). In addition, Zhang et al. (14) emphasized the importance of the Notch pathway in ATC (69\% of patients involved), particularly the involvement of NOTCH2NL, suggesting that it may influence cancer progression through the Notch pathway.

Different alleles of NOTCH2NL enhance the ability of the Notch pathway with varying potentials. Notch 2 N-terminal like C (NOTCH2NLC) and Notch $2 \mathrm{~N}$-terminal like $\mathrm{R}(\mathrm{NOTCH} 2 \mathrm{NLR})$ are considered non-functional pseudogenes, and NOTCH2NLA and Notch $2 \mathrm{~N}$-terminal like B $(N O T C H 2 N L B)$ have a stable number of combined copies, while their ability to enhance $\mathrm{NOTCH} 2$ activation to promote the proliferation of nerve cells is similar (11). At the same time, some studies have confirmed that NOTCH2NL, as a new type of NOTCH-like gene, can be encapsulated by vesicles, and then be transported out of cells to bind to NOTCH receptors and ligands, regulate Notch signaling pathways (26), and may affect chromodomain helicase DNA binding protein 1 like (CHD1L) expression (11). The CHD1L experiment confirmed that it is significantly related to the metastasis process of ovarian cancer (27). The expression of CHD1L protein detected by IHC can be used as a new prognostic biomarker for patients with ovarian cancer. Therefore, NOTCH2NL may influence the occurrence and development of OC through these mechanisms, and the specific influence mechanism should be explored in future work.

\section{Limitations and strengths}

There are some limitations to the current study. First, the activation of EMT is associated with multiple signaling pathways, such as Notch, Wnt, and transforming growth factor- $\beta$ (TGF- $\beta$ ) pathways (28). However, the association between NOTCH2NL, Notch signaling, or other pathways, and EMT needs to be fully elucidated. Second, considering the regional specificity of NOTCH2NL expression in the tumors of patients, our data did not demonstrate a differential expression of NOTCH2NL in different stages and histological types of human OC tissues, nor verify its ability to promote cancer progression in different OC cell lines. Third, the current study did not conduct validation experiments in the eight-gene prognostic model except for NOTCH2NLA in OC.

This study had several strengths. Strikingly, neither the regulation of EMT by NOTCH2NLA nor its oncogenic role has been previously reported in OC. Thus, our study increases our understanding of NOTCH2NLA function and suggests its potential role in cancer progression. Second, our study is the first to use in vivo and in vitro assays combined with bioinformatics to explore the function of NOTCH2NL, while previous research focused on its copy number variations and mutations. Moreover, the current study separately analyzed the significance of NOTCH2NLA in OC to refine the role of NOTCH2NL.

\section{Conclusions}

In conclusion, we established an eight-gene prognostic model for OC. Our study highlights that NOTCH2NLA silencing may inhibit proliferation, migration, and invasion in OC, and its high expression promotes solid tumors and induces EMT in xenograft tumors. Taken together, these data provide novel insights into the mechanisms of OC mediated by NOTCH2NLA.

\section{Acknowledgments}

We extend our sincere thanks to Zhe Wang and Wenhao Wang from Second Hospital of Shanxi Medical University for helping with experimental techniques and proposals. Funding: This study was supported by the National Natural Science Foundation of China (No. 81702583) and the Natural Science Foundation of Shanxi Province (No. 201901D111366). 


\section{Footnote}

Reporting Checklist: The authors have completed the ARRIVE reporting checklist. Available at https://dx.doi. org/10.21037/atm-21-5435

Data Sharing Statement: Available at https://dx.doi. org/10.21037/atm-21-5435

Conflicts of Interest: All authors have completed the ICMJE uniform disclosure form (available at https://dx.doi. org/10.21037/atm-21-5435). The authors have no conflicts of interest to declare.

Ethical Statement: The authors are accountable for all aspects of the work in ensuring that questions related to the accuracy or integrity of any part of the work are appropriately investigated and resolved. The study was conducted in accordance with the Declaration of Helsinki (as revised in 2013). Experiments were performed under a project license (No. SYDL2021007) granted by the animal ethics board of Shanxi Medical University, in compliance with the Ministry of Science and Technology of China guidelines for the care and use of animals.

Open Access Statement: This is an Open Access article distributed in accordance with the Creative Commons Attribution-NonCommercial-NoDerivs 4.0 International License (CC BY-NC-ND 4.0), which permits the noncommercial replication and distribution of the article with the strict proviso that no changes or edits are made and the original work is properly cited (including links to both the formal publication through the relevant DOI and the license). See: https://creativecommons.org/licenses/by-nc-nd/4.0/.

\section{References}

1. Sung H, Ferlay J, Siegel RL, et al. Global Cancer Statistics 2020: GLOBOCAN Estimates of Incidence and Mortality Worldwide for 36 Cancers in 185 Countries. CA Cancer J Clin 2021;71:209-49.

2. He R, Zhu B, Liu J, et al. Women's cancers in China: a spatio-temporal epidemiology analysis. BMC Womens Health 2021;21:116.

3. Sun H, Yan L, Chen H, et al. Development of a nomogram to predict prognosis in ovarian cancer: a SEER-based study. Transl Cancer Res 2020;9:5829-42.

4. Chen W, Zheng R, Baade PD, et al. Cancer statistics in
China, 2015. CA Cancer J Clin 2016;66:115-32.

5. Hultgren NW, Fang JS, Ziegler ME, et al. Slug regulates the Dll4-Notch-VEGFR2 axis to control endothelial cell activation and angiogenesis. Nat Commun 2020;11:5400.

6. Huang CC, Cheng $\mathrm{SH}, \mathrm{Wu} \mathrm{CH}$, et al. Delta-like 1 homologue promotes tumorigenesis and epithelialmesenchymal transition of ovarian high-grade serous carcinoma through activation of Notch signaling. Oncogene 2019;38:3201-15.

7. Liu Z, Zhu Y, Li F, et al. GATA1-regulated JAG1 promotes ovarian cancer progression by activating Notch signal pathway. Protoplasma 2020;257:901-10.

8. Muñoz-Galván S, Felipe-Abrio B, García-Carrasco M, et al. New markers for human ovarian cancer that link platinum resistance to the cancer stem cell phenotype and define new therapeutic combinations and diagnostic tools. J Exp Clin Cancer Res 2019;38:234.

9. Perez-Fidalgo JA, Ortega B, Simon S, et al. NOTCH signalling in ovarian cancer angiogenesis. Ann Transl Med 2020;8:1705.

10. Yang M, Zhang G, Wang Y, et al. Tumour-associated neutrophils orchestrate intratumoural IL-8-driven immune evasion through Jagged2 activation in ovarian cancer. $\mathrm{Br} \mathrm{J}$ Cancer 2020;123:1404-16.

11. Fiddes IT, Lodewijk GA, Mooring M, et al. HumanSpecific NOTCH2NL Genes Affect Notch Signaling and Cortical Neurogenesis. Cell 2018;173:1356-1369.e22.

12. Killela PJ, Pirozzi CJ, Reitman ZJ, et al. The genetic landscape of anaplastic astrocytoma. Oncotarget 2014;5:1452-7.

13. Liao L, Ji X, Ge M, et al. Characterization of genetic alterations in brain metastases from non-small cell lung cancer. FEBS Open Bio 2018;8:1544-52.

14. Zhang L, Ren Z, Su Z, et al. Novel Recurrent Altered Genes in Chinese Patients With Anaplastic Thyroid Cancer. J Clin Endocrinol Metab 2021;106:988-98.

15. Sala-Gaston J, Martinez-Martinez A, Pedrazza L, et al. HERC Ubiquitin Ligases in Cancer. Cancers (Basel) 2020;12:1653.

16. Beizavi Z, Zohouri M, Asadipour M, et al. IL-27, a pleiotropic cytokine for fine-tuning the immune response in cancer. Int Rev Immunol 2021;40:319-29.

17. Wang WD, Wu GY, Bai KH, et al. A prognostic stemness biomarker CCDC80 reveals acquired drug resistance and immune infiltration in colorectal cancer. Clin Transl Med 2020;10:e225.

18. Mukherjee A, Huynh V, Gaines K, et al. Targeting the High-Mobility Group Box 3 Protein Sensitizes 
Chemoresistant Ovarian Cancer Cells to Cisplatin. Cancer Res 2019;79:3185-91.

19. Millstein J, Budden T, Goode EL, et al. Prognostic gene expression signature for high-grade serous ovarian cancer. Ann Oncol 2020;31:1240-50.

20. Yan S, Fang J, Chen Y, et al. Comprehensive analysis of prognostic gene signatures based on immune infiltration of ovarian cancer. BMC Cancer 2020;20:1205.

21. Shi Z, Zhao Q, Lv B, et al. Identification of biomarkers complementary to homologous recombination deficiency for improving the clinical outcome of ovarian serous cystadenocarcinoma. Clin Transl Med 2021;11:e399.

22. Zheng M, Mullikin H, Hester A, et al. Development and Validation of a Novel 11-Gene Prognostic Model for Serous Ovarian Carcinomas Based on Lipid Metabolism Expression Profile. Int J Mol Sci 2020;21:9169.

23. Pan HX, Luo GN, Wan SQ, et al. Detection of de novo genetic variants in Mayer-Rokitansky-Küster-Hauser syndrome by whole genome sequencing. Eur J Obstet Gynecol Reprod Biol X 2019;4:100089.

Cite this article as: Guo W, Sun Z, Zhao N, Zhou Y, Ren J, Huang L, Ping Y. NOTCH2NLA silencing inhibits ovarian carcinoma progression and oncogenic activity in vivo and in vitro. Ann Transl Med 2021;9(22):1669. doi: 10.21037/atm$21-5435$
24. Funato K, Smith RC, Saito Y, et al. Dissecting the impact of regional identity and the oncogenic role of humanspecific NOTCH2NL in an hESC model of H3.3G34Rmutant glioma. Cell Stem Cell 2021;28:894-905.e7.

25. Suzuki IK, Gacquer D, Van Heurck R, et al. HumanSpecific NOTCH2NL Genes Expand Cortical Neurogenesis through Delta/Notch Regulation. Cell 2018;173:1370-1384.e16.

26. Heide M, Huttner WB. Human-Specific Genes, Cortical Progenitor Cells, and Microcephaly. Cells 2021;10:1209.

27. He WP, Zhou J, Cai MY, et al. CHD1L protein is overexpressed in human ovarian carcinomas and is a novel predictive biomarker for patients survival. BMC Cancer 2012;12:437.

28. Lin YT, Wu KJ. Epigenetic regulation of epithelialmesenchymal transition: focusing on hypoxia and TGF- $\beta$ signaling. J Biomed Sci 2020;27:39.

(English Language Editor: B. Draper) 